\title{
Tongue-Base Suspension in Conjunction with Uvulopalatopharyngoplasty for Treatment of Severe Obstructive Sleep Apnea: Long-Term Follow-Up Results
}

Eugenio Vicente, MD; José M. Marín, MD; Santiago Carrizo, MD; M. J. Naya, MD

Objectives/Hypothesis: To evaluate the usefulness of tongue-base suspension (TBS) in addition to uvulopalatopharyngoplasty (UPPP) in the treatment of obstructive sleep apnea syndrome (OSAS). Study Design: Long-term prospective case series. Setting: University tertiary care medical center. Methods: Fifty-five consecutive patients with severe OSAS (apnea-hypopnea index [AHI] $52.8 \pm 14.9$ events/hr) with multilevel upper airway obstruction who refused treatment with continuous positive airway pressure underwent UPPP plus TBS with the Repose system. All patients were evaluated before surgery by clinical history, Epworth Sleepiness Scale (ESS), fiberoptic nasopharyngoscopy with Müller maneuver, and nocturnal polysomnography (PSG). After 3 years of surgery, all patients were re-evaluated at the clinic and had ESS test and full PSG. Surgical success was defined when the ESS dropped bellow 11, and the AHI decreased below the threshold of 20 events per hour of sleep and at least $50 \%$ from the preoperative value Results: There were 42 (78\%) patients in which the AHI score decreased more than 50\%, the AHI was lower than 20 events/hr in the PSG, and the ESS was lower than 11 after 3-years of follow-up (surgical success index). Logistic regression analysis demonstrated that body mass index at baseline was the only variable with significant statistical power to predict surgical success (odds ratio $0.85 ; 0.73-0.95$, 95\% confidence interval, $P<.01)$ Conclusion: When associated with UPPP, the TBS technique performed with the Repose system demonstrates a surgical success of up to $\mathbf{7 8 \%}$ for patients with

From the ENT (E.V., M.J.N.) and Respiratory (J.M.M., s.c.) Departments, Hospital Universitario Miguel Servet, Zaragoza, Spain. $15,2006$.

Editor's Note: This Manuscript was accepted for publication March

Send correspondence to Dr. Eugenio Vicente, ORL Service, Hospital Universitario Miguel Servet, Avda Isabel la Católica 1-3, 50009-Zaragoza, Spain. E-mail: evicenteg@seorl.net.

DOI: 10.1097/01.mlg.0000224498.09015.d9 severe OSA who refused nasal continuous positive airway pressure. Key Words: Tongue-base suspension, uvulopalatopharyngoplasty, obstructive sleep apnea syndrome.

Laryngoscope, 116:1223-1227, 2006

\section{INTRODUCTION}

Obstructive sleep apnea (OSA) is characterized by repetitive episodes of total (apnea) or partial (hypopnea) pharyngeal collapse during sleep. To restore pharyngeal airflow, patients with OSA have recurrent arousals from sleep that are associated with sleep fragmentation and activation of sympathetic nervous system. As a consequence, patients may suffer from daytime sleepiness and neurocognitive and cardiovascular consequences. ${ }^{1,2}$ Nasal continuous positive airway pressure (CPAP) is the treatment of choice in severe OSA; nevertheless, the therapy is cumbersome, and compliance is poor. Surgery is used as an alternative to CPAP to correct anatomic abnormalities or just because of patient choice. Because in OSA narrowing of the upper airway occurs at the oropharynx and hypopharynx levels, most of the surgical procedures are directed to increase the airway patency at those sites. Uvulopalatopharyngoplasty (UPPP) is used as palatal surgery, whereas surgical treatment of hypopharynx collapse includes many procedures, most of them associated with significant morbidity. ${ }^{3}$ Recently, preliminary results with a minimally invasive technique for tongue-base suspension (TBS) have been described: the so-called Repose System. ${ }^{4-7}$ Nevertheless, these series included small numbers of patients and did not take into consideration daytime symptoms, ${ }^{4,6}$ the TBS was performed without UPPP, ,,5,7 and the follow-up time was short in all reports. The aim of our study was to evaluate the long-term usefulness of the Repose System in a large group of patients with severe OSA with multilevel airway obstruction. 


\section{PATIENTS AND METHODS}

\section{Patients}

As part of our protocol for the treatment of severe OSA in our sleep clinic, surgery is offered as an alternative to all patients who did not tolerate or reject therapy with nasal CPAP. We prospectively observed consecutive patients with severe OSA treated with the Repose System. Inclusion criteria were 1) persistent symptoms of OSA; 2) apnea-hypopnea index (AHI) greater than 30 as determined by polysomnography (PSG); 3) nasal CPAP intolerance or refusal, and 4) obstruction at both the oropharynx and hypopharynx levels. Exclusion criteria were 1) age older than 70 years; 2 ) severe obesity as defined by a body mass index (BMI) greater than $40 \mathrm{~kg} / \mathrm{m} 2$, and 3) cardiac or respiratory failure. The ethics committee of our institution approved the study, and all participants gave their written informed consent. The authors have no financial interests in the Repose System for TBS.

\section{Clinical Evaluation}

As described previously, all patients had a comprehensive clinical history including completion of a specific sleep questionnaire. ${ }^{8}$ Daytime sleepiness was estimated by the Epworth Sleepiness Scale (ESS) to rate hypersomnolence 0 to $24 .^{9}$ Physical examination included measurement of BMI and neck circumference. Sleep evaluation was performed by full PSG. All-night PSG studies monitored the following variables: electroencephalogram, electrooculogram, chin and leg electromyogram, electrocardiogram, nasal and oral airflow measures, thoracic and abdominal efforts, and pulse oximetry. The variables analyzed were AHI, lowest $\mathrm{SaO}_{2}$, and the percentage of sleep time with a $\mathrm{SaO}_{2}$ below 90\% (CT90).

\section{Presurgical Evaluation}

Physical examination of the upper airway was supplemented by nasopharyngolaryngoscopy with the Muller maneuver technique and with lateral cephalometric radiographs. Patients were classified as having oropharyngeal obstruction when there is an elongation of the soft palate or when the soft palate collapses against the pharyngeal wall with the Muller maneuver. Hypopharyngeal obstruction was defined as when the base of the tongue collapses against the posterior/lateral pharyngeal wall or when there is a narrow posterior airway space on cephalometric analysis. All candidates demonstrated greater than 50\% collapse at the levels of the base of the tongue and the soft palate. Patients with oropharyngeal obstruction received surgical palate and patients with hypopharyngeal obstruction received TBS with the Repose System.

\section{Surgical Techniques}

Surgery was performed under general anesthesia, and patients were monitored the first postoperative day in the intensive care unit. During the surgical session, nasal reconstruction was performed if obstructive nasal deformities were present. UPPP was performed using the technique described by Simmons et al. ${ }^{10}$ The details of the surgical procedure for the TBS using the Repose System (Influence, Inc. San Francisco, CA) have been extensively describe elsewhere, ${ }^{4}$ and it was performed following the instructions for use included in the surgical kit.

\section{Postoperative Evaluation and Follow-up}

In this study, we have included patients operated during the inclusion period lasting from January 2001 to December 2002 and with a minimum postoperative follow-up of 3 years. All patients were evaluated at the clinic using ESS to check daytime sleepiness and PSG to monitor sleep disorders 3 months after surgery and then 3 years later. Outcomes were mortality, operative and postoperative morbidity, and AHI and ESS score. Success was defined when the ESS droped bellow 11 and the AHI decreased below the threshold of 20 events per hour of sleep and at least $50 \%$ from the preoperative value.

\section{Statistical Analysis}

All data were analyzed using SPSS version 10.0 (SPSS, Chicago, IL) and are presented as mean \pm SD. The Wilcoxon's rank sum test was used to compare the preoperative and postoperative results. Logistic multiregression analysis models were used to assess predicted factor for postsurgical success as defined previously. Significance was accepted for $P<.05$.

\section{RESULTS}

There were 1,233 consecutive patients referred to our sleep unit between January 2001 and December 2002 for evaluation of suspected sleep apnea. Of these, $581 \mathrm{had}$ severe OSA, and nasal CPAP was recommended as the treatment of choice. There were 122 patients who did not accept this therapy for various reasons or showed very bad compliance (less than $2 \mathrm{hr}$ use per night). To all of these consecutive patients, surgery as an alternative therapeutic approach was proposed. The patients' baseline characteristics are listed in Table I. There were no differences in baseline clinical and sleep data among patients who did or did not accept the surgical approach. All patients were habitual and heavy snorers and reported excessive day-

TABLE I.

Baseline Characteristics.

\begin{tabular}{lccc}
\hline Measurements & $\begin{array}{c}\text { Patients Accepted Surgery } \\
(\mathrm{n}=55)\end{array}$ & $\begin{array}{c}\text { Patients Rejected Surgery } \\
(\mathrm{n}=67)\end{array}$ & $P$ Value \\
\hline Ratio male:female & $51: 4$ & $58: 9$ & .381 \\
Age, years & $47.3 \pm 4.5$ & $48.1 \pm 3.7$ & .058 \\
BMI, kg/m & $29.6 \pm 4.8$ & $30.1 \pm 3.8$ & .522 \\
Neck circumference, cm & $41.2 \pm 3.4$ & $41.4 \pm 3.2$ & .739 \\
ESS & $12.2 \pm 3.3$ & $12.8 \pm 3.1$ & .303 \\
AHI, events per hour & $52.8 \pm 14.9$ & $54.1 \pm 13.5$ & .614 \\
CT90, \% & $28.5 \pm 18.6$ & $29.6 \pm 17.4$ & .736 \\
Lowest O2 Sat, \% & $76.2 \pm 12.4$ & $75.5 \pm 11.2$ & .744 \\
\hline \hline
\end{tabular}

$\mathrm{BMI}=$ body mass index; ESS = Epworth Sleepiness Scale; $\mathrm{AHI}=$ apnea-hypopnea index; $\mathrm{CT} 90$ = percentage of time with oxyhemoglobin saturation below $90 \%$. 
time sleepiness. Mean baseline PSG data were consistent with severe OSA syndrome, showing a high AHI (52.8 \pm 14.9) and severe oxygen desaturation as reflected by a high CT90 (28.5 \pm 18.6).

Among the 55 patients who underwent UPPP plus TBS using the Repose System, there were no significant perioperative complications such as postoperative bleeding, need for tracheotomy, or uncontrollable pain. All patients received routine antibiotics, analgesics, and postoperative re-educational rehabilitation of speech. In 21 patients, septoplasty was caused by obstructive nasal deformities, and in 17 patients, tonsillectomy was also performed.

In Table II are presented the clinical and PSG variables at baseline and at 3 year follow-up of 54 patients because 1 was lost to follow-up when he moved overseas. BMI and neck circumference did not change. All other variables improved in every patient undergoing UPPP-TBS. There were $42(78 \%)$ patients in which the AHI score decreased more than $50 \%$, the AHI was lower than 20 events/hour in PSG, and the ESS was lower than 11 after 3 years of follow-up (surgical success index). The AHI mean value for the entire group decreased from $52.4 \pm 14.9$ to $14.1 \pm 23.5(P<.001)$ (Fig. $1)$. Logistic regression analysis was used to obtain an odds ratio for surgical success. BMI at baseline was the only variable with significant statistical power to enter into the model and showed an odds ratio of $0.85(0.73-$ $0.95,95 \%$ confidence interval, $P<.01$ ). Indeed, the relationship of increased presurgery BMI and decreased postsurgery AHI showed an inverse and significant relationship (Fig. 2). The subgroup of patients who had septoplasty in addition to TBS plus UPPP did not have different outcomes than those who did not have nasal reconstruction. In comparison with patients who were successfully treated with UPPP-TBS, those who were not successfully treated had at baseline a higher BMI $(P=.01)$ and bigger neck circumference $(P=.03)$, but there were no significant differences between these two subgroups in age or EES or PSG variables.

\section{DISCUSSION}

Treatment of OSA is aimed at reducing the number of episodes of apnea-hypopnea, the number of arousals, and to normalized the oxyhemoglobin saturation level. These changes have been correlated with an improvement in daytime alertness and quality of life. The mainstay of therapy for OSA syndrome is nasal CPAP, which maintains a patent airway during sleep, thereby avoiding apnea. However, although CPAP is highly effective, compliance and acceptance with the treatment is a problem. Surgical treatment for OSA is also available. The most used surgical therapy, UPPP, was described by Fujita et al. ${ }^{11}$ in 1981 ; only $41 \%$ of patients who undergo the procedure obtain an AHI of less than 20 events per hour, which is not judged an adequate surgical outcome. More aggressive surgery such as UPPP with genioglossal advancement, maxillomandibular advancement, hyoid advancement, or hyoidthyroidpexia has been reported. ${ }^{3}$ The reported decrease in AHI ranged from $10 \%$ to $60 \%$; however, most patients in these series had mild or moderate OSA, and follow-up was short.

Our study includes a large series of patients with severe OSA, and, in a controlled fashion, the follow-up was extended at least for 3 years. We showed that UPPP-TBS was effective in $78 \%$ of patients with severe OSA. This rate of success is higher than in previous studies, in which the rate of success ranged between $25 \%$ and $70 \%$ using UPPP in addition with other surgical techniques. ${ }^{12-15}$ In all those series, patients had mild to severe OSA, and the success rate was predicted by the AHI.

Definition of surgical success has been widely varyiable. In most of the reports, success has been considered when there was a reduction of the AHI score below 20 events per hour or when there was a decrease of $50 \%$ in this parameter after surgery. Nasal CPAP improves dramatically daytime symptoms of patients with OSA, and this is considered as good response to the therapy. In the same way, to considerer a surgical technique as successful, the operated patients must report a significant improvement in daytime sleepiness (e.g., reducing the EES

TABLE II.

Changes in Clinical and Polysomnographic Variables 3 Years after Surgery.

\begin{tabular}{lccc}
\hline Measurements & $\begin{array}{c}\text { Presurgery } \\
(\mathrm{n}=55)\end{array}$ & $\begin{array}{c}\text { Postsurgery } \\
(\mathrm{n}=54)\end{array}$ & $P$ Value \\
\hline Ratio male:female & $51: 4$ & $50: 4$ & .388 \\
Age, years & $47.3 \pm 4.5$ & $50.2 \pm 4.7$ & $<.01$ \\
BMI, kg/m² & $29.6 \pm 4.8$ & $28.1 \pm 4.8$ & .105 \\
Neck circumference, cm & $41.2 \pm 3.4$ & $41.1 \pm 3.5$ & .880 \\
ESS & $12.2 \pm 3.3$ & $8.2 \pm 6.1$ & .002 \\
AHI, events per hour & $52.8 \pm 14.9$ & $14.1 \pm 23.5$ & $<.001$ \\
CT90, \% & $28.5 \pm 18.6$ & $5.1 \pm 12.4$ & $<.001$ \\
Lowest O2 Sat, \% & $76.2 \pm 12.4$ & $82.2 \pm 11.2$ & .009 \\
Change in AHI > 50\% + postsurgery AHI $<$ & & $42(78)$ & \\
20, $\mathrm{n}$ (\%) (postsurgery success) & & & \\
\hline \hline
\end{tabular}

BMI = body mass index; ESS = Epworth Sleepiness Scale; AHI = apnea-hypopnea index; CT90 = percentage of time with oxyhemoglobin saturation below $90 \%$. 

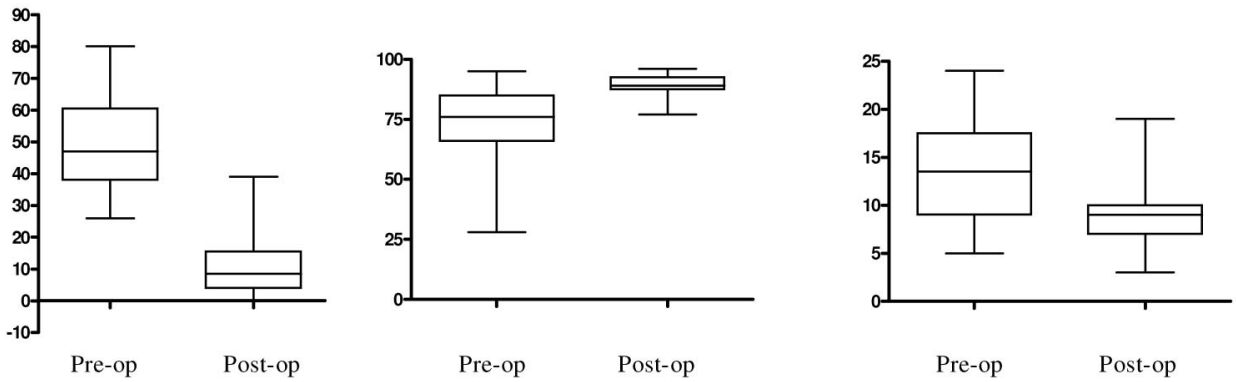

Fig. 1. Box plots of the apnea-hypopnea index, lowest $\mathrm{O} 2$ saturation, and Epworth sleepiness scale at baseline (preoperative) and 3 years after surgery (postoperative). Centerline of box denotes median, extremes of box interquartile range, and bars upper and lower of $95 \%$ of data. For significance of preoperative versus postoperative, see Table II.

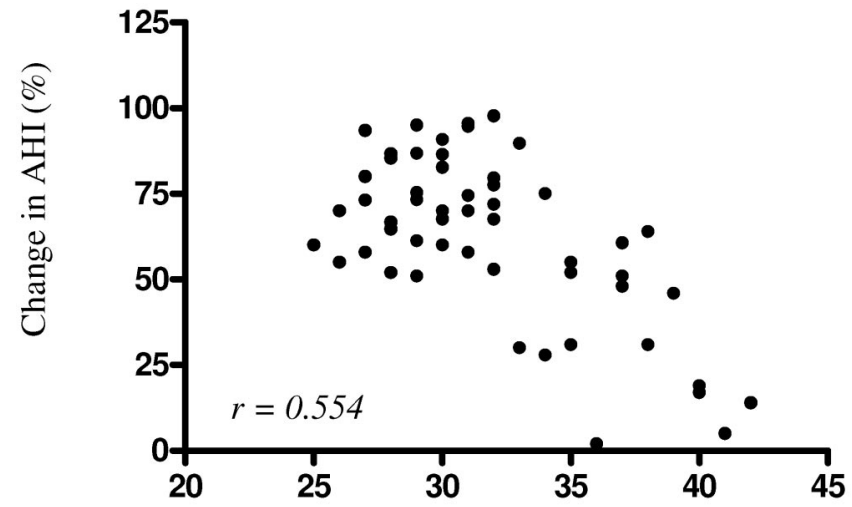

Body Mass Index $\left(\mathrm{Kg} / \mathrm{m}^{2}\right)$

Fig. 2. Relationship between presurgery baseline body mass index and postsurgery change in apnea-hypopnea index after 3 years follow-up. $(r=0.554, P<.001)$.

below 10). Our definition of surgical success included improvement in both variables: AHI and EES. That is, in the success group, patients remain free of daytime symptoms and showed an improvement in PSG data, as stated above. This makes our findings even stronger because, if only the change in AHI was included in the definition, then the surgical success rate of our study would have been greater than the current $78 \%$. One important finding of our study was the negative correlation between the presurgery BMI and the postsurgery AHI. The more obese patients (e.g., BMI $>35 \mathrm{~kg} / \mathrm{m}^{2}$ ) were those who had a lower decrease in the postsurgical AHI. It is feasible that the increase intraluminal pharyngeal pressure was not overcome by surgery in this type of patient. Because we did not measure this pressure before and after surgery, we will need to investigate the cause for the poor response in very obese patients.

All of our patients were operated on because they rejected nasal CPAP and were found to have preoperative obstruction in both the oropharynx and the hypopharynx. Therefore, we designed a multilevel surgical approach using UPPP and TBS to remove the obstruction. UPPP was performed with the classic technique, ${ }^{10}$ and TBS was performed with the Repose System ${ }^{4}$ during the same surgical session. There was a previous study that also used this approach, but it was performed with a short series of patients $(\mathrm{n}=19)$, the analysis was retrospective, and the follow-up period was brief (mean $=3.8 \mathrm{mo}$ ). In this study, a $46 \%$ reduction in the preoperative versus the final postoperative AHI was demonstrated. ${ }^{6}$ Our results are superior because of our large series or bias selection of patients really motivated for a surgical therapy of their OSA syndrome.

\section{CONCLUSIONS}

This study was conducted to assess prospectively the role of the Repose System in the long-term management of patients with OSA with multilevel airway obstruction. When associated with UPPP, the Repose System demonstrates a surgical success of up to $78 \%$ for patients with severe OSA (more than 30 apnea-hypopnea events per hour of sleep) who refused to use nasal CPAP. We defined surgical success as when the postsurgical AHI decreased below 20 events per hour or by more than $50 \%$ and when the ESS index decreased below 11 after 3 years of followup. The best response was obtained in patients whose BMI was lower than $35 \mathrm{~kg} / \mathrm{m}^{2}$.

\section{BIBLIOGRAPHY}

1. Kim HC, Young T, Matthews CG et al. Sleep-disordered breathing and neuropsychological deficits: a populationbased study. Am J Respir Crit Care Med 1997;156: 1813-1819.

2. Shahar E, Whitney CW, Redline S et al. Sleep-disordered breathing and cardiovascular disease: cross-sectional results of the Sleep Heart Health Study. Am J Respir Crit Care Med 2001;163:19-25.

3. Riley RW, Powell NB, Guilleminault C. Obstructive sleep apnea syndrome: a review of 306 consecutively treated surgical patients. Otolaryngol Head Neck Surg 1993;108: $117-125$.

4. DeRowe A, Gunther E, Fibbi A et al. Tongue-base suspension with a soft tissue-to-bone anchor for obstructive sleep apnea: preliminary clinical results of a new minimally invasive technique. Otolaryngol Head Neck Surg 2000;122: 100-103.

5. Woodson BT. A tongue suspension suture for obstructive sleep apnea and snorers. Otolaryngol Head Neck Surg 
2001;124:297-303.

6. Miller FR, Watson D, Malis D. Role of the tongue base suspension suture with The Repose System bone screw in the multilevel surgical management of obstructive sleep apnea. Otolaryngol Head Neck Surg 2002;126:392-398.

7. Kuhnel TS, Schurr C, Wagner B et al. Morphological changes of the posterior airway space after tongue base suspension. Laryngoscope 2005;115:475-480.

8. Marin JM, Carrizo SJ, Vicente E et al. Long-term cardiovascular outcomes in men with obstructive sleep apneahypopnea with or without treatment with continuous positive airway pressure: an observational study. Lancet 2005; 365:1046-1053.

9. Johns MW. A new method for measuring daytime sleepiness: the Epworth Sleepiness Scale. Sleep 1991;14:540-545.

10. Simmons FB, Guilleminault C, Silvestri R. Snoring and some obstructive sleep apnea can be cured by oropharyngeal surgery: palatopharyngoplasty. Arch Otolaryngol 1983; 109:503-507.
11. Fujita S, Conway W, Zorick F, Roth T. Surgical correction of anatomic abnormalities in obstructive sleep apnea syndrome: uvulopalatopharyngoplasty. Otolaryngol Head Neck Surg 1981;89:923-934.

12. Ramirez S, Loube DI. Inferior sagittal osteotomy with hyoid bone suspension for obese patients with sleep apnea. Arch Otolaringol Head Neck Surg 1996;122:953-957.

13. Battega G, Pepin JL, Veale D et al. Obstructive sleep apnea syndrome: fifty-one consecutive patients treated by maxillofacial surgery. Am J Respir Crit Care Med 2000;162: 641-649.

14. Johnson NT, Chinn J. Uvulopalatopharyngoplasty and inferior sagittal mandibular osteotomy with genioglossus advancement for treatment of obstructive sleep apnea. Chest 1994;105:278-283.

15. Yao M, Utley DS, Terris DJ. Cephalometrics parameters after multilevel pharyngeal surgery for patient with obstructive sleep apnea. Laryngoscope 1998;108:789-795. 